\title{
Towards robust biomarkers of psychosocial interventions
}

\author{
Nadia Micali ${ }^{1,2} \cdot$ Cristina Berchio $^{3,4}$ \\ Published online: 25 January 2019 \\ (c) Springer-Verlag GmbH Germany, part of Springer Nature 2019
}

At-risk adolescents, i.e. those youth experiencing a range of externalizing behaviours, including conduct and antisocial problems, and living in adverse socio-economic environments, are an understudied population. In this issue, Pincham and colleagues [1] examined the neural impact of a psychosocial intervention in at-risk adolescents using eventrelated potentials as biomarkers of therapeutic change. The hypothesis is that a psychosocial intervention program may improve neural and behavioural functioning.

At-risk youth have a tendency to act impulsively and select immediate recompenses even if potentially associated with negative consequences. In this study, neural determinants of decision-making were assessed in two groups of adolescents receiving a psychosocial intervention, using a modified version of the Taylor Aggression Paradigm during event-related potentials (ERPs) recording. As an index of affective and feedback brain functioning, the authors investigated the late positive potential (LPP), the feedbackrelated negativity (FRN), and the P300. Two groups were compared: youth who had had the psychosocial intervention for $\leq 4$ months and those who had had the same intervention for $\geq 9$ months.

Findings indicated that during the decisional phase the minimally exposed group showed reduced LPP responses to provocation and penalized the adversaries more harshly than adolescents exposed to intervention for a longer period. These findings are suggestive of a greater sensitivity to

Nadia Micali

nadia.micali@unige.ch

1 Child and Adolescent Psychiatry Division, Department of Pediatrics, Gyneacology and Obstetrics, Geneva University Hospital, Geneva, Switzerland

2 Department of Psychiatry, Faculty of Medicine, University of Geneva, Geneva, Switzerland

3 Department of Basic Neurosciences, University of Geneva, Geneva, Switzerland

4 Mood Disorders Unit, Service of Psychiatric Specialties, Department of Mental Health and Psychiatry, University Hospitals of Geneva, Geneva, Switzerland social provocation in the minimally exposed group, likely due to immature brain decision-making strategies.

Participants minimally exposed to intervention also revealed diminished FNR and P300 responses to feedback signals compared with those who received the intervention for longer. These results are suggestive of feedback hyposensitivity for the minimally exposed intervention group. Taken together, these data demonstrate that a psychosocial intervention is associated with neural and behavioural improvements and reduction of externalizing symptoms.

The study is limited by the lack of a longitudinal and/ or controlled design and a well-defined intervention. Nevertheless, it is very interesting thanks to its ability to 'take the lab into the community'. Highlighting the possibility of using ERPs as a solid marker of change for therapeutic interventions that might be difficult to test could enable using a robust methodology to assess change following a psychosocial intervention.

Neural determinants of risk-taking behaviour can be assessed using ERPs. Robust findings demonstrate an association between the LPP and the development of emotional regulation strategies [2-4]. Maturational changes of the brain reward system are believed to be responsible for risktaking behaviours during adolescence [5]. Therefore, ERPs related to reward processing may provide complementary information on brain function and risk-taking in adolescence. The FRN is a negative wave observed after approximately $200 \mathrm{~ms}$ after the appearance of signals concerning individuals' performance [6]. Evidence suggests that risktaking behaviours in adolescents are associated with FRN abnormalities [7]. Activity of the P300 indexes cognitiveattentional functioning [8], and is linked to behavioural inhibition [9], and development of aggression in humans [10]. Reduced P300 amplitudes in adolescence are predictive of antisocial behaviours in adulthood [11].

The present study also supports the notion that P300 and FNR can reflect immature responses to feedback processing and externalizing problems and, therefore, predict high risk for adverse developmental outcomes. The ability to describe brain function with high temporal and spatial resolution, 
using electrical neuroimaging procedures [12], can aid gaining an understanding of the neural correlates of developmental problems and psychiatric disorders at relatively low cost, with high portability, and can be very useful for hardto-reach populations and young research subjects.

Studies on the effect of psychosocial interventions assessing ERPs patterns are lacking, especially during development. Further studies may shed new light on neural circuits and related abnormalities that might be amenable to change in at-risk youth and, thus, pave the way to new interventions.

\section{References}

1. Pincham HL, Bryce D, Fonagy P, Fearon RMP (2018) Psychosocial intervention in at-risk adolescents: using event-related potentials to assess changes in decision making and feedback processing. Eur Child Adolesc Psychiatry

2. Dennis TA, Hajcak G (2009) The late positive potential: a neurophysiological marker for emotion regulation in children. J Child Psychol Psychiatry 50(11):1373-1383

3. Babkirk S, Rios V, Dennis TA (2015) The late positive potential predicts emotion regulation strategy use in school-aged children concurrently and two years later. Dev Sci 18(5):832-841

4. Bunford N, Kujawa A, Swain JE, Fitzgerald KD, Monk CS, Phan KL (2017) Attenuated neural reactivity to happy faces is associated with rule breaking and social problems in anxious youth. Eur Child Adolesc Psychiatry 26(2):215-230

5. Galvan A (2010) Adolescent development of the reward system. Front Hum Neurosci 4:6

6. Hauser TU, Iannaccone R, Stämpfli P, Drechsler R, Brandeis D, Walitza $S$ et al (2014) The feedback-related negativity (FRN) revisited: new insights into the localization, meaning and network organization. NeuroImage 84:159-168

7. Crowley MJ, Wu J, Crutcher C, Bailey CA, Lejuez C, Mayes LC (2009) Risk-taking and the feedback negativity response to loss among at-risk adolescents. Dev Neurosci 31(1-2):137-148

8. Cui T, Wang PP, Liu S, Zhang X (2017) P300 amplitude and latency in autism spectrum disorder: a meta-analysis. Eur Child Adolesc Psychiatry 26(2):177-190

9. Iacono WG, Malone SM, McGue M (2003) Substance use disorders, externalizing psychopathology, and P300 event-related potential amplitude. Int J Psychophysiol 48(2):147-178

10. Petersen IT, Hoyniak CP, Bates JE, Staples AD, Molfese DL (2018) A longitudinal, within-person investigation of the association between the P3 ERP component and externalizing behavior problems in young children. J Child Psychol Psychiatry 59(10):1044-1051

11. Yoon HH, Malone SM, Burwell SJ, Bernat EM, Iacono WG (2013) Association between P3 event-related potential amplitude and externalizing disorders: a time-domain and time-frequency investigation of 29-year-old adults. Psychophysiology 50(7):595-609

12. Michel CM, Murray MM (2012) Towards the utilization of EEG as a brain imaging tool. NeuroImage 61(2):371-385 